\title{
On the VLSI Implementation of Adaptive-Frequency Hopf Oscillator
}

\author{
Arash Ahmadi, Member, IEEE, Eduardo Mangieri, Koushik Maharatna, Member, IEEE, \\ Srinandan Dasmahapatra, and Mark Zwolinski, Senior Member, IEEE
}

\begin{abstract}
In this paper, a new VLSI implementable Hopf oscillator with dynamic plasticity is proposed for next-generation portable signal processing application. A circuit-realizable piece-wise linear function has been used to govern the frequency adaptation characteristic of the proposed oscillator. Furthermore, a straightforward method is suggested to extract the frequency component of the input signal. Mathematical model of the oscillator is derived and it is shown, using VHDL-AMS model, that despite using a new nonlinear function, the oscillator exhibits the same characteristics and learning behavior as the original one with improved learning time. Subsequently, an equivalent circuit model and transistor level implementation for the oscillator is suggested and the mathematical model is confirmed with system and circuit level simulations. Capability of such oscillator to extract frequency futures without doing explicit signal processing is shown with examples of both synthetic and real-life EMG signals.
\end{abstract}

Index Terms-Adaptive oscillator, analog signal processing, Hopf oscillator, nonlinear oscillator.

\section{INTRODUCTION}

D ESPITE impressive progresses in current VLSI, fundamental issues such as: power consumption, process variation, soft errors, noise, and other non-idealities in nanometer process technologies threaten to nullify the great achievements of scaling that the semiconductor industry has come to expect [1]. This indicates that advances in manufacturing technology alone cannot address all the problems effectively. Therefore, material and device advances must be complemented by innovations in system level and new computational paradigms to enrich or go beyond the standard computation models and architectures.

From this perspective, nature, especially biology, can be a rich source of inspiration to engineers for designing new types of information processing systems. The animals' brain, for instance, is far more efficient than CMOS technologies. Neural pattern generators control the locomotion of animals, which make them able to adapt their body movement dynamically

Manuscript received February 11, 2010; revised June 10, 2010 and August 25, 2010; accepted October 21, 2010. Date of publication December 30, 2010; date of current version April 27, 2011. This paper was recommended by Associate Editor S. Callegari.

A. Ahmadi is with the Electronic Department, School of Engineering, Razi University, Kermanshah, 67149, Iran (e-mail: aahmadi70@gmail.com).

E. Mangieri, K. Maharatna, S. Dasmahapatra, and M. Zwolinski are with the School of Electronics and Computer Science, University of Southampton, Southampton, SO17 IBJ, U.K.

Color versions of one or more of the figures in this paper are available online at http://ieeexplore.ieee.org.

Digital Object Identifier 10.1109/TCSI.2010.2092070 with the changing signals from environment and manage to coordinate multiple degrees of freedom needed for locomotion. This can be done only by using signals of the right frequencies, phases, and amplitudes in response to the external affects. This mechanism includes a wide range of periodic behaviors in the nature from a single cell movement to complex locomotion systems such as different types of walking, running, flying, swimming, etc. These mechanisms mostly rely on periodic behavior of the oscillators and their capability of synchronization with the applied external signals.

Inspired by natural computational systems, nonlinear oscillator networks have been a subject of intensive researches, ranging from mathematical models to practical applications. Their wide range of applications and capabilities have made them a serious suggestion for the future system design in electronics [2]. Although oscillators are very well known in all their varieties, they are traditionally designed to have a fixed intrinsic frequency. This "fixed intrinsic frequency" results in two basic limitations, which have restricted their application in aforementioned emerging fields. First is synchronization limit, which means, commonly oscillators have narrow synchronization ranges. Second, they have no memory of their past interactions. This means that they return to their intrinsic frequency of oscillations if the external signal to which they were synchronized vanishes. Consequently, in applications that require wide-range synchronization and memory of past interactions, traditionally designed oscillators are not suitable. In this case, the main alternative to the traditional oscillators are nonlinear adaptive oscillators.

Theoretically, nonlinear oscillators has been used for decades to model diverse natural phenomena such as neuronal signaling models [3], Central Pattern Generation (CPG) [4], [5], associative memory [6], [7] and beat perception [8], [9]; to engineering applications such as universal machines [10], [11], image processing [12], logic computation, [13] and robotics [14], [15]. The main challenge, which has limited a generalized practical application of nonlinear oscillators, is the mathematical complexity inherent in their model. This is a serious problem, which is preventing their application in real-life VLSI systems.

Hopf dynamic system, as a nonlinear oscillator, has found specific applications in artificial locomotion systems and basic frequency analysis. Recently, in [15] and [16], Buchli et al. introduced a new learning rule in the Hopf oscillator [17], according to which the intrinsic frequency of the system evolves towards the frequency of an external perturbation and thus incorporates dynamic plasticity in the oscillator itself. The novelty of this work is that the oscillator is able to extract the frequency 
of any input signal without any explicit signal processing. This work shows an immense application potential of the proposed oscillatory system in developing future generations of signal processing circuits and systems. The present work is primarily motivated by this work in which we look at the nonlinear oscillator described in [16] from a practical VLSI circuit design point of view, so that it can be exploited for developing advanced and portable signal processing systems for a variety of applications. More precisely, in this paper we consider the possibility of its efficient hardware realization in analog circuit domain. A possible digital implementation of such a system will be discussed in our future work.

In terms of VLSI realization, the oscillator proposed in [16] has several fundamental difficulties as follows.

1) The learning rule introduced utilizes terms like $y / \sqrt{x^{2}+y^{2}}$ and $x^{2}+y^{2}$ which are extremely difficult to realize in practice.

2) The oscillation frequency, $\omega$, is considered as a system state which is directly dependent on the input signal. In an analog equivalent, this state value should be realized as a capacitance voltage (or inductance current), if targeted to VLSI implementation. In practice, this linear dependence of $\omega$ on capacitor voltage restricts its range of adaptation to only a few volts (or equivalently to a few Hertz, a small oscillation-frequency basin).

3) The frequency convergence/learning time of this oscillator is very high (several thousand seconds in some cases). In a practical application this delay is not acceptable.

4) Although the proposed oscillator can "learn" the frequency of the external signal, the extraction of its amplitude requires a large number of coupled oscillators (1000) which is very costly in terms of real hardware particularly for a signal with a wide frequency spectrum.

These four practical problems inspired us to develop a novel and simple VLSI realizable model while staying within the framework proposed in [16]. To the best of our knowledge, this is the first time such a circuit has been proposed. Here, firstly, we use a simple circuit realizable piecewise-linear function to govern the frequency adaptation or learning process. We show that the proposed function not only produces the oscillation characteristics described in [16] but also adapts the frequency at a faster rate. Secondly, we develop a new method for extending the frequency basin of this modified oscillator. Subsequently, we develop a generic circuit design methodology for the entire system and validate it by circuit simulation. Thirdly, we introduce a new and simple methodology and subsequent circuit that is capable of finding the relative amplitudes of the input signal harmonics.

Some parts of this study have been reported in [18] in a short form, but here we present a detailed analysis on the limit cycle characteristics and nature of the convergence for the proposed oscillator. We have also proposed a new nonlinear function which is a modification to that presented in [18] to make the proposed oscillator more implementation friendly compared to those in [16] and [18]. In addition a novel method for amplitude extraction of the constituent frequency components with their corresponding system/circuit level simulation results are presented.
The rest of the paper is organized as follows: Section II presents the background of Hopf oscillator, [17], and the modification done to it as described in [16]. In Section III, we propose a new adaptive frequency non-linear oscillator. Its circuit level implementation and corresponding simulation results are provided in Sections IV and V, respectively. General discussion about the open questions and conclusions are drawn in Sections VI and VII.

\section{BACKGROUND}

The dynamics of the Hopf oscillator can be described by the following ordinary differential equations [17]:

$$
\begin{aligned}
& \dot{x}=\left(\mu^{2}-\left(x^{2}+y^{2}\right)\right) x+\omega y \\
& \dot{y}=\left(\mu^{2}-\left(x^{2}+y^{2}\right)\right) y-\omega x
\end{aligned}
$$

where $\mu^{2}>0, x, y \in R$ are the states of the oscillator, $\omega$ is the oscillation frequency and $\mu$ determines the steady state amplitude of oscillation $\left(x_{\infty}^{2}+y_{\infty}^{2}=\mu^{2}\right)$ [19].

Under these conditions (1) gives stable periodic solutions, known as limit cycles, [17]

$$
x=\mu \sin \left(\omega t+\theta_{0}\right), \quad y=\mu \cos \left(\omega t+\theta_{0}\right)
$$

where $\theta_{0}$ is determined by the initial conditions. This solution represents a circle of the form $x^{2}+y^{2}=\mu^{2}$, around the origin of the state space.

In the Hopf oscillator, the oscillation radius is fixed and independent of the oscillation frequency. Changes to the phase of the oscillation, however, can be permanent and happen only if affect the limit cycle in the direction tangential to the oscillation, when represented in the state space. The perpendicular component of the perturbation force is damped out in the long term leaving the tangential component of the perturbation (typically termed as the "teaching force") to tune the limit cycle frequency to one of its frequency components. More details can be found in [20].

Exploiting the above mentioned property, in [16], the Hopf oscillator described in (1) is modified to incorporate dynamic plasticity under an additive perturbation $I(t)$ as shown in (3)

$$
\begin{aligned}
& \dot{x}=\left(\mu^{2}-\left(x^{2}+y^{2}\right)\right) x+\omega y+K I(t) \\
& \dot{y}=\left(\mu^{2}-\left(x^{2}+y^{2}\right)\right) y-\omega x \\
& \dot{\omega}=K I(t) \frac{y}{\sqrt{x^{2}+y^{2}}}
\end{aligned}
$$

where $K>0$ is a coupling constant.

Physically, in (3), the term $\mu$ represents the radius of the oscillation circle and the term $\sin (\phi)=y / \sqrt{x^{2}+y^{2}}$ represents the teaching force in the state space at the point $(x(t), y(t))$ as shown in Fig. 1. As it is explained in [20], this dynamic system creates a limit cycle oscillation which synchronize with the frequency of the input perturbation, $I(t)$. The oscillator will have a tendency to accelerate or decelerate, according to the tangential component of the $I(t)$ in the phase plane, which on average results in an oscillation at a frequency of the $I(t)$. This 


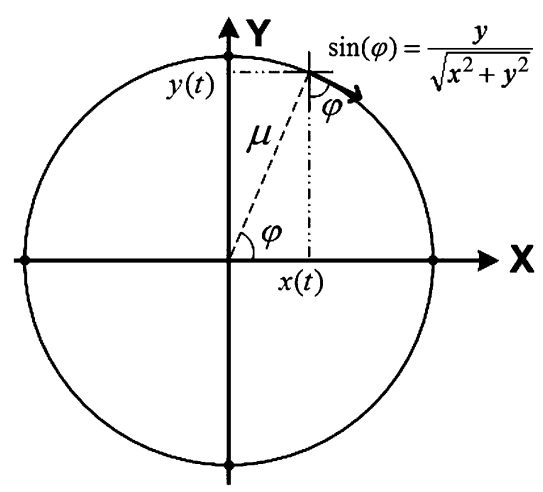

Fig. 1. State-space representation of the oscillation characteristics of the oscillator in [16].

teaching force, however, in frequency domain is manifested as a jitter around the converged frequency. If $I(t)$ comprises different frequencies with different amplitudes, depending on the initial values of the state variables, the oscillator tunes itself to the frequency component having the highest amplitude. More importantly, even when $I(t)$ is withdrawn, the oscillator keeps oscillating with the frequency it becomes tuned to. Thus, a number of such oscillators may be employed to obtain the complete time-frequency map of $I(t)$, which is a fundamental operation in signal processing. More detailed description of different properties of this adaptive frequency oscillator can be found in [15], [16], and [20].

\section{PROPOSED OSCILLATOR}

\section{A. Mathematical Modification of Hopf Oscillator}

Let us consider the equations in (1). Both of them consist of two parts: $\left(\mu^{2}-\left(x^{2}+y^{2}\right)\right) x$ and $+\omega y$; in the first equation and $\left(\mu^{2}-\left(x^{2}+y^{2}\right)\right) y$ and $-\omega x$ in the second one. The second part in these equations is responsible for providing feedback in the system to create its oscillatory behavior. The first part, on the other hand, is responsible for the amplitude limitation of the oscillation by comparing a measure of the oscillation amplitude or energy $\left(x^{2}+y^{2}\right)$ with a limit value $\left(\mu^{2}\right)$. We note that, in effect, the frequency behavior of such an oscillatory system depends only on the sign of $\dot{x}$ and $\dot{y}$ and the relative values of $x$ and $y$ to $\mu$. From this understanding of the system behavior, we propose to change the first parts of the both equations in (1) as

$$
\begin{aligned}
& \dot{x}=(\mu-(|x|+|y|)) \operatorname{sgn}(x)+\omega y \\
& \dot{y}=(\mu-(|x|+|y|)) \operatorname{sgn}(y)-\omega x
\end{aligned}
$$

where $|x|+|y|$ is the new measure for the oscillation amplitude/ energy and $\operatorname{sgn}(\cdot)$ is the sign value of $x$ and $y$. Comparing with the oscillator presented in [18], two multipliers in the equation are replaced by sign functions, which has a very simple physical realization in analog circuit design (a comparator). The statespace representation of (4) is shown in Fig. 2.. It can be seen that in this representation the circular function in (1) and Fig. 1 is replaced by a square shaped function $|x|+|y|=\mu$, which is easily realizable in practice.

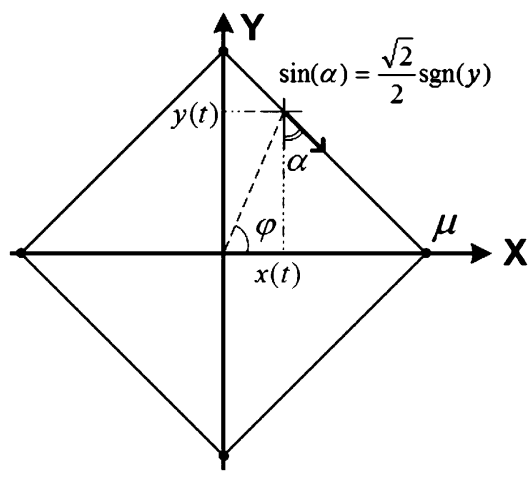

Fig. 2. State-space representation of the oscillation characteristics of the proposed oscillator.

\section{B. Stability and Limit Cycle Behavior of the Modified Oscillator}

The stability of the proposed modified Hopf oscillator system can be determined using the Lyapunov second method with the energy function as [21]

$$
V(x, y)=\frac{1}{2}\left(x^{2}+y^{2}\right)
$$

where, by differentiating $V$ and replacing $x$ and $y$ from (4), we have

$$
\begin{aligned}
\dot{V} & =x \dot{x}+y \dot{y} \\
& =(\mu-(|x|+|y|))(|x|+|y|)
\end{aligned}
$$

resulting in:

$$
\begin{cases}\dot{V}>0 & |x|+|y|<\mu \\ \dot{V}=0 & |x|+|y|=\mu \\ \dot{V}<0 & |x|+|y|>\mu\end{cases}
$$

which means that the system has a stable limit cycle for

$$
|x|+|y|=\mu
$$

The inequalities of (7) apply to our applications in which initial values are always bounded and therefore, the system is guided to a limit cycle state.

To derive the oscillation behavior of the system, by transforming (4) to the polar coordinates $(x=r \cos (\phi)$ and $y=$ $r \sin (\phi))$, we get

$$
\begin{aligned}
\dot{r}=(\mu-r & (|\cos \phi|+|\sin \phi|))(\sin \phi+\cos \phi) \\
\dot{\phi}=\omega+\frac{1}{r} & (\mu-r(|\sin \phi|+|\cos \phi|) \\
& \quad \times(\operatorname{sgn}(\sin \phi) \cos \phi-\operatorname{sgn}(\cos \phi) \sin \phi)) .
\end{aligned}
$$

Applying the steady-state criterion with $\dot{r}=0$, we get

$$
\left\{\begin{array}{l}
r=\frac{\mu}{|\sin \phi|+|\cos \phi|} \\
\dot{\phi}=\omega
\end{array}\right.
$$

Thus the steady state oscillation can be described as

$$
x_{s s}(t)=\frac{\mu \sin (\omega t)}{|\sin (\omega t)|+|\cos (\omega t)|}
$$




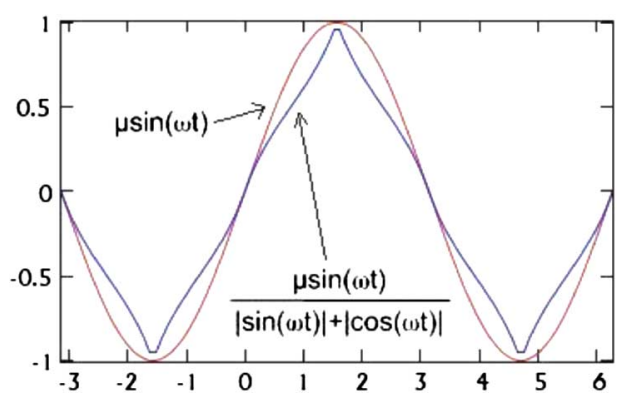

Fig. 3. Output signal of the modified oscillator comparing with monotone sinusoidal oscillation.

assuming $\phi(0)=0$. The nature of the oscillation described in (11) is depicted in Fig. 3 comparing with $\mu \sin (\omega t)$ (as in the original Hopf oscillator).

From Fig. 3, the important thing to note is that the shape of the oscillation waveform resembles a triangular wave rather than a pure sinusoid. However, the frequency characteristic and amplitude value of it are exactly similar to that of the reference sinusoid which is in conformation of the state-space representation of the proposed oscillator as shown in Fig. 2.

\section{New Adaptive Frequency Hopf Oscillator}

The most important implication of the modification proposed above is the change it makes in the frequency adaptation equation in (3). From a geometrical viewpoint, as shown in Fig. 2, in our proposed oscillator, to create the limit cycle behavior of the system, the circular function $x^{2}+y^{2}=\mu^{2}$ is replaced with the square shaped function $|x|+|y|=\mu$, thus referring to Fig. 2, noting that the gradient of each side of the square is $45^{\circ}$, under an additive perturbation the tangential component of the teaching force can simply be defined as $\sin (\alpha)=$ $(\sqrt{2} / 2) \cdot \operatorname{sgn}(y)$.

Using these modifications in (3), the dynamics of the proposed oscillator can be described by the following equation:

$$
\begin{aligned}
& \dot{x}=(\mu-(|x|+|y|)) \operatorname{sgn}(x)+\omega y+K I(t) \\
& \dot{y}=(\mu-(|x|+|y|)) \operatorname{sgn}(y)-\omega x \\
& \dot{\omega}=K I(t) \cdot \operatorname{sgn}(y)
\end{aligned}
$$

assuming that $\sqrt{2} / 2$ in the third equation is included in the coupling parameter $K$.

Knowing that $\operatorname{sgn}(y)=y /|y|$ for $y \neq 0$ and $|y|=\sqrt{y^{2}}$, the adaptation equation can be rewritten as

$$
\dot{\omega}=K I(t) \cdot \operatorname{sgn}(y)=K I(t) \cdot \frac{y}{\sqrt{y^{2}}}
$$

Comparing with the adaptation equation in (3), we observe

$$
\operatorname{sgn}(y)=\frac{y}{\sqrt{y^{2}}} \geq \frac{y}{\sqrt{x^{2}+y^{2}}} .
$$

Using this basic inequality to compare the third equations in (12) and (3), which are responsible for the frequency adaptation mechanism of the oscillators; it is evident that the rate of change of $\omega$ in the proposed oscillator is larger than the rate of change of $\omega$ in the original Hopf oscillator. This results in a faster learning dynamics in the modified oscillator.

One important aspect of the proposed oscillator is to ensure its frequency convergence. According to the argument presented in [20], due to the stable nature of the Hopf oscillator and since the learning dynamics only depends on the conjugate symmetry of the complex Fourier series of the input signal, using any combination of periodic functions as the learning rule will not change the frequency convergence characteristics. This can also be proved analytically following the perturbation based approach outlined in [20]. Thus, for brevity we omit that proof here. However, in Section III-F through numerical simulation we will show that the proposed oscillator indeed follows the frequency convergence property of the oscillator in [16].

\section{Extension of the Frequency Basin}

As mentioned earlier, from the practical VLSI circuit design point of view, one fundamental problem of the oscillator in [16] is that due to the linear relationship between $\omega$ and the voltage representing its value, the achievable size of the frequency basin is very small for any practical purposes. To overcome this problem, we observe from (12)

$$
\dot{\omega}=K I(t) \cdot \operatorname{sgn}(y(t))
$$

and after integration

$$
\begin{aligned}
\omega & =\omega(0)+K \int_{0}^{t} I(\tau) \operatorname{sgn}(y(\tau)) d \tau, \\
& =\omega_{0}+\Delta \omega
\end{aligned}
$$

assuming $\omega(0)=\omega_{0}$. In this way we split the oscillation frequency into two parts $\omega_{0}$ and $\Delta \omega$. The first part is the intrinsic frequency of the oscillator and the latter is a variable part which evolves to synchronize the oscillator with the input signal. Using this modification in (12), the final governing equation for our oscillator can be given as

$$
\begin{aligned}
\dot{x} & =(\mu-(|x|+|y|)) \operatorname{sgn}(x)+\omega_{0} y+\Delta \omega \cdot y+K I(t) \\
\dot{y} & =(\mu-(|x|+|y|)) \operatorname{sgn}(y)-\omega_{0} x-\Delta \omega \cdot x \\
\dot{\Delta \omega} & =K I(t) \cdot \operatorname{sgn}(y) \quad \text { with } \Delta \omega(0)=0 .
\end{aligned}
$$

In this way, a spectrum analyzer can be designed using a set of oscillators in which their intrinsic frequencies $\left(\omega_{0}\right)$ are chosen to be equally distributed over the range of the expected spectrum of the input signal. Every oscillator in this set will tune itself to the frequency component having the largest amplitude among those included in the frequency basin around $\omega_{0}$. A detailed analysis of the choice of $\omega_{0}$ to cover a particular range of the frequency spectrum is provided in [20], which can also be applied here without any loss of generality. 


\section{E. Computing the Relative Amplitude of the Frequency Component}

Considering the Fourier expansion of the input perturbation $I(t)=\sum_{i=0}^{N}\left(a_{i} \sin \omega_{i} t+b_{i} \cos \omega_{i} t\right)=\sum_{i=0}^{N} A_{i} \sin \left(\omega_{i} t+\theta_{i}\right)$, analyzing frequency spectrum of the input also requires information about the magnitude of each of the frequency components $\left(A_{i}\right)$. Recalling the basic mathematical methods for computing the Fourier series based on orthogonality of $\sin (\cdot)$ and $\cos (\cdot)$, theoretically, the amplitudes $a_{i}, b_{i}$ or $A_{i}$ of the frequency component $\omega_{i}$ can be calculated as:

$$
\begin{aligned}
a_{i} & =\frac{2}{T} \int_{0}^{T} \sin \left(\frac{2 \pi i}{T} \tau\right) I(\tau) d \tau \\
b_{i} & =\frac{2}{T} \int_{0}^{T} \cos \left(\frac{2 \pi i}{T} \tau\right) I(\tau) d \tau
\end{aligned}
$$

where $T$ is the period of the first harmonic [22], [23].

In our case, after the oscillator learning time, by which it converges to a single frequency of the input perturbation (when $t \rightarrow \infty$ then $x(t) \rightarrow \mu \sin \left(\omega_{i} t\right)$ and $y(t) \rightarrow \mu \cos \left(\omega_{i} t\right)$ ), using the above equation, we can conclude that

$$
\begin{aligned}
a_{i} & \propto \frac{1}{T} \int_{0}^{T} x(\tau) I(\tau) d \tau \\
b_{i} & \propto \frac{1}{T} \int_{0}^{T} y(\tau) I(\tau) d \tau \\
A_{i} & \propto \frac{1}{T} \frac{1}{\sin \theta_{i}} \int_{0}^{T} x(\tau) I(\tau) d \tau \\
& \propto \frac{1}{T} \frac{1}{\cos \theta_{i}} \int_{0}^{T} y(\tau) I(\tau) d \tau
\end{aligned}
$$

where the right-hand side of the equations represent averaging operation over time $T$. This proportionality means that after the oscillator's convergence to a frequency component in $I(t)\left(\omega_{i}\right.$ for instance), the amplitude of this frequency component $\left(A_{i}\right)$ will be proportional to the time-average of $x(\tau) \cdot I(\tau)$. In terms of system dynamics, this relationship can be represented as an extra state equation in (17) as

$$
\dot{A}=K_{A} I(t) \cdot x .
$$

This equation results in a linear relationship between $A_{i}$ and the integration time, creating an ever-increasing signal in the circuit. However, in practice it is possible to extract the slope of the integration result within a limited time (i.e. after the oscillator's convergence) and stop the integration thereafter. The calculated slopes correspond to the comparative amplitudes of different frequency components of the input signal. Simulation results for this method are presented in the next subsection.

\section{F. The Complete Oscillator and System Level Simulation}

As mentioned earlier, in this work we consider that the state variables are represented by capacitor voltages. With this as- sumption, combining (17) with (20), the complete governing dynamics of the proposed adaptive frequency oscillator can be given as follows:

$$
\begin{aligned}
\dot{V}_{x}= & \left(\mu-\left(\left|V_{x}\right|+\left|V_{y}\right|\right)\right) \operatorname{sgn}\left(V_{x}\right)+\omega_{0} V_{y} \\
& +V_{\Delta \omega} V_{y}+K V_{i n}(t) \\
\dot{V}_{y}= & \left(\mu-\left(\left|V_{x}\right|+\left|V_{y}\right|\right)\right) \operatorname{sgn}\left(V_{y}\right)-\omega_{0} V_{x}-V_{\Delta \omega} V_{x} \\
\dot{V}_{\Delta \omega}= & K V_{i n}(t) \cdot \operatorname{sgn}\left(V_{y}\right) \quad \text { with } V_{\Delta \omega}(0)=0 \\
\dot{V}_{A}= & K_{A} V_{i n}(t) \cdot V_{x}
\end{aligned}
$$

where $V_{x}, V_{y}, V_{\Delta \omega}$, and $V_{A}$ represent the state variables in terms of voltages representing $x, y, \Delta \omega$ and amplitude $(A)$ respectively.

To investigate the functional behavior of the proposed oscillator, it was modeled using VHDL-AMS [24] and simulated by Mentor Graphics AdvanceMS tools. At the same time, for comparison, the oscillator proposed in [16] was also implemented. Three issues were investigated, namely, the rate of frequency convergence of the proposed oscillator, effect of $K$ on the frequency convergence rate, and the tuning property of an array of oscillators having different intrinsic frequencies to their nearby frequencies.

Fig. 4 shows the frequency convergence characteristic of the proposed oscillator along with the oscillator proposed in [16] for a single sinusoid with $10 \mathrm{~Hz}$ frequency when $K$ is set to 10 . It can be clearly seen that the proposed oscillator converges to the desired frequency faster than that proposed in [16], as predicted by (14). But the convergence characteristics of the oscillator proposed in [16] is smoother than the one proposed here which shows some "roughness". This is because the value of the tangential teaching component of the adopted piece-wise linear function regulating the oscillator has a fixed value for each particular region in the phase space. On the other hand, the same component for the nonlinear function governing the oscillator proposed in [16] adjusts itself smoothly in the phase space. However, this behavior does not affect the final value of the frequency at which the oscillator converges. To be noted that here we present a system level simulation of the oscillators started from $\omega_{0}=0$, therefore, despite long learning time of these simulation results, in the circuit implementation of the oscillator it is shown that convergence time is considerably lower (see Section V).

Fig. 5 shows the frequency convergence characteristics of the proposed oscillator for different values of $K$ keeping the initial values of the state variables constant. It is evident that with increasing values of $K$ the oscillator converges to the desired frequency at faster rate. However, our simulation shows that a bigger value of $K$ can cause oscillatory behavior in $\omega$ after convergence, which in real life can be considered similar to a jitter around the converged frequency. Therefore, a tradeoff between the speed and stability has to be made for choosing the value of $K$ under a particular circumstance.

Fig. 6 briefly compares the frequency jitters of two different versions of implementable oscillators (presented in [18] and here). It is observable from figures that oscillators presented here and in [18] have a similar jitter pattern. Jitter values are also calculated in Table I for these oscillators. The calculated 


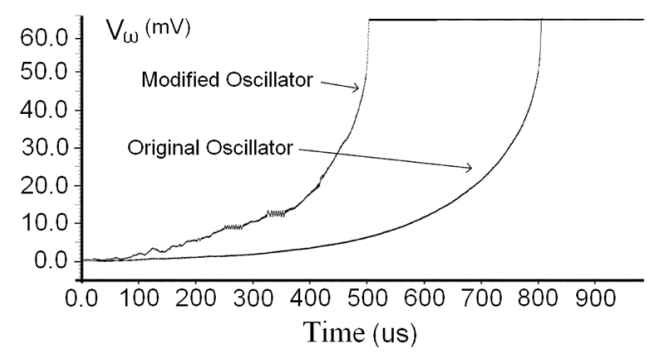

Fig. 4. Frequency convergence comparison between the oscillator in [16] and the proposed one, $(f=10, K=10)$.

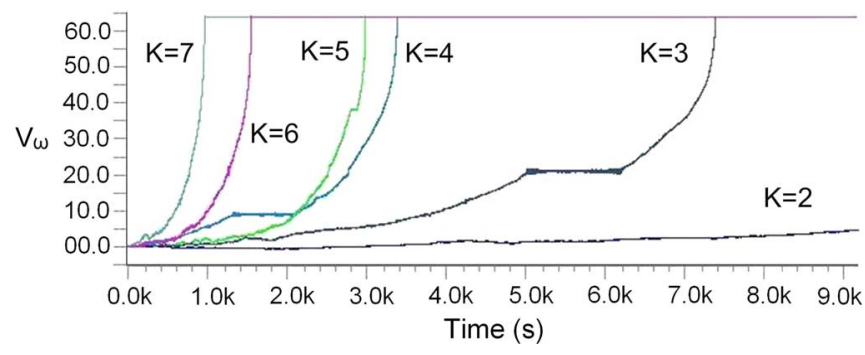

Fig. 5. Frequency convergence characteristics for different values of $K$ ( $f=$ $20 \pi, \mathrm{K}=2-7)$.

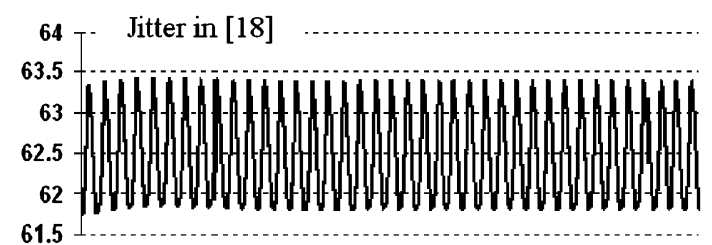

(a)

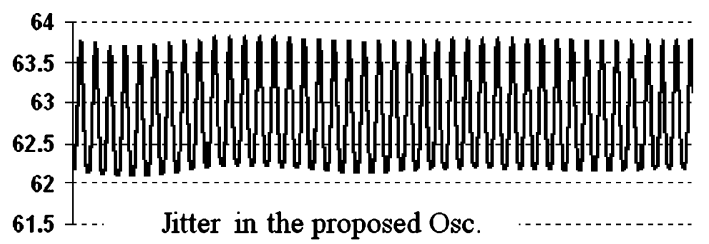

(b)

Fig. 6. Jitters comparison after frequency convergence between the oscillators in a) $[18]$ and $b)$ the proposed here, $(f=10, K=10)$.

TABLE I

JiTTER VALUES FOR DIFFERENT VERSIONS OF THE HOPF OSCILLATOR ( $\mathrm{f}=10, \mathrm{~K}=10$ )

\begin{tabular}{|l||l|l|}
\hline \hline Parameter & Ref. [18] & Proposed Osc. \\
\hline \hline Jitter P2P & 1.703196678 & 1.726628386 \\
\hline Jitter P2P (\%) & 2.726180294 & 2.748146212 \\
\hline Jitter RMS & 0.321973064 & 0.333826579 \\
\hline Jitter RMS (\%) & 0.515358346 & 0.531326981 \\
\hline Learning Time & $0.5(\mathrm{~ms})$ & $0.5(\mathrm{~ms})$ \\
\hline \hline
\end{tabular}

values in the Table I show that, in terms of jitter and convergence, differences between oscillators in [18] and our present work are negligible.

As it is shown above, this jitter exists in both (original and proposed) oscillators due to the nature of the adaptation mechanism which is based on acceleration and decelerations of the oscillatory limit cycle induced by $I(t)$ frequency components. The

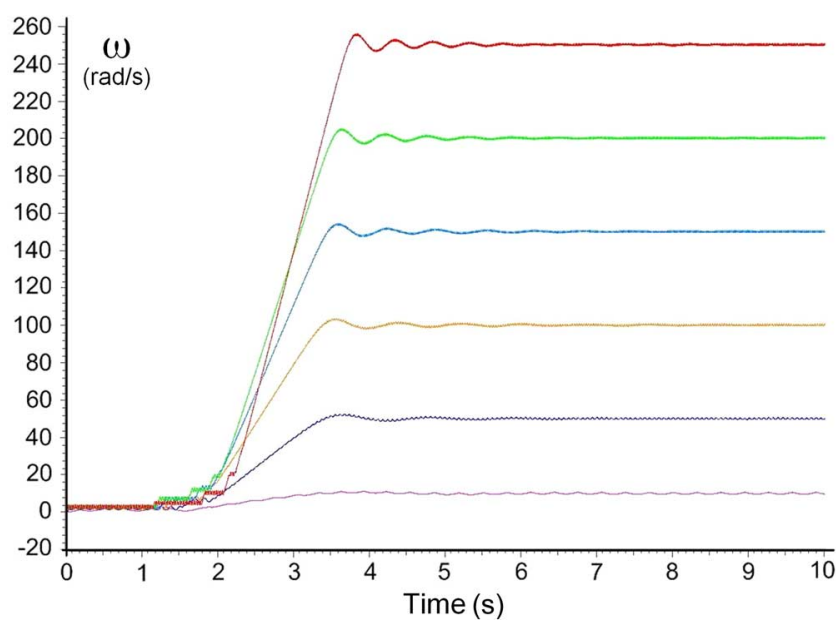

Fig. 7. Frequency convergence characteristics for different input frequencies.

only difference arises from the fact that the teaching forces [tangential component of $\mathrm{I}(\mathrm{t})$ ], are different between these two oscillators. The teaching force in (3) changes smoothly when the oscillation point moves from one quadrant to another in the phase plane (see Fig. 1), where this change is more stepwise in ours (see Fig. 2). Although this effect is reflected in learning behavior of the oscillator where the proposed oscillator exhibits faster convergence rate, after convergence, however, system continues to oscillate with the learned frequency and effects of adaptation mechanism and teaching force will be eliminated or reduced considerably. Therefore, we would not expect significant differences between the original oscillator and the proposed one after frequency convergence. Our comparative system level simulation of the oscillators supports this perception.

Fig. 7 shows oscillator response to different input frequencies. It is observable that there is a one to one linear dependency between different frequencies the oscillator can adapt and the state value of $\omega$.

Fig. 8 shows the frequency adaptation behavior of the oscillator under a nonstationary external perturbation. In this simulation, an input signal with different frequencies and amplitudes at different time instants is used. As it is shown, the oscillator "learns" different frequencies of the input at different time slices. This characteristic can be employed to perform a time-frequency analysis of a nonstationary input signal by using an array of oscillators operating at different "time zones" (equivalent to the Wavelet transform). Also note that when the input signal is withdrawn, the oscillator keeps oscillating with the latest frequency it encountered in the input signal, thereby "memorizing" it.

To demonstrate the behavior of our proposed oscillator in separating different frequency components present in a particular signal, we have used an input signal composed of three frequencies, viz. 50, 30, and $10 \mathrm{~Hz}$, each of the components having different amplitudes. We employed identical oscillators with different initial state values of $\omega_{0}$. The result is shown in Fig. 9. In this case, each of these oscillators converges to its nearest frequency value and keeps oscillating at that frequency when the input signal is withdrawn. It is to be noted that for each of the oscillators, the choice of initial value of $\omega$ impacts significantly 

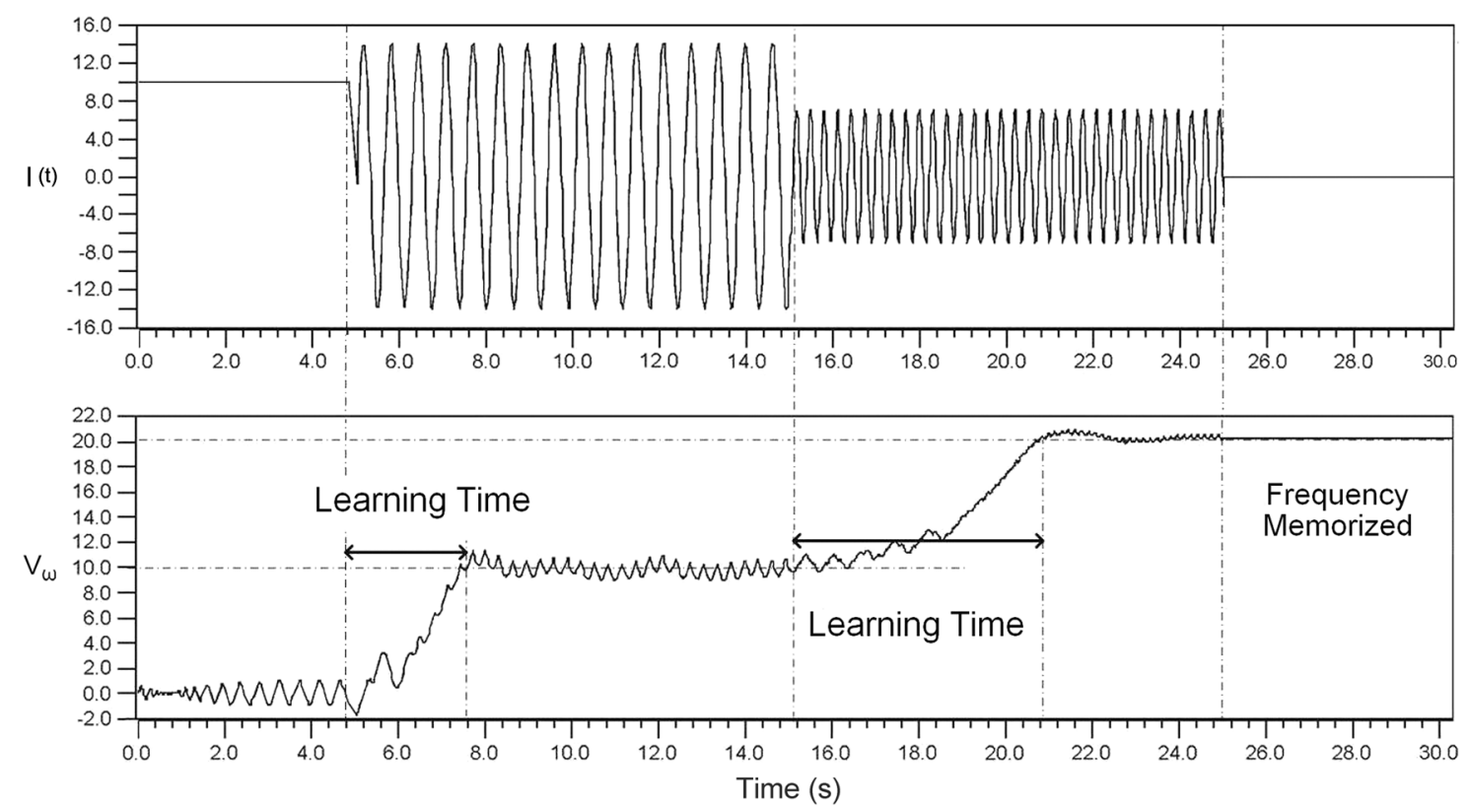

Fig. 8. Oscillator response to a nonstationary input signal.
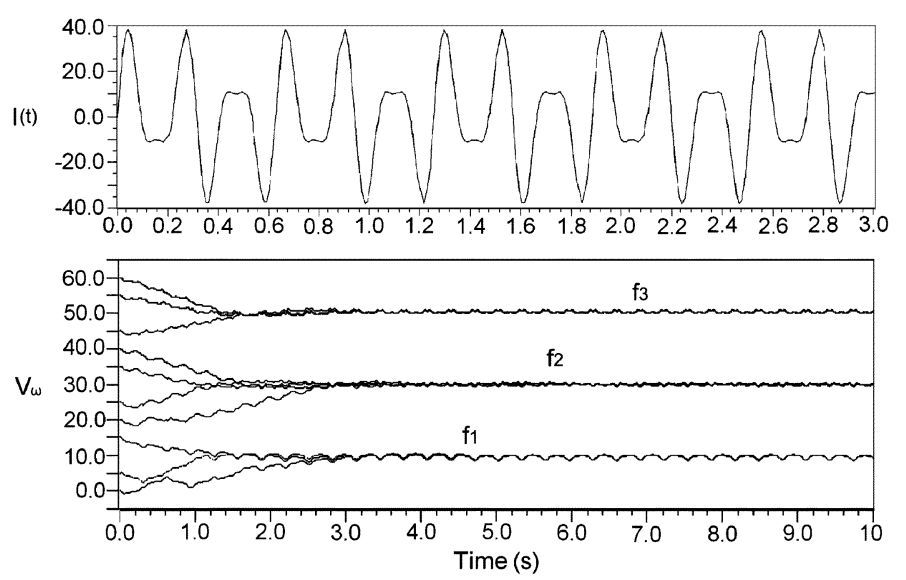

Fig. 9. Array of similar oscillators with different initial values. Each oscillator converges to a frequency components of the $\mathrm{I}(\mathrm{t})$ depending on its initial $\omega, I(t)=12 \sin (50 t)+27 \sin (30 t)+5 \sin (10 t)$.

on the time of frequency convergence which is also shown in Fig. 9.. Fig. 9 also confirms that each value of $\omega_{0}$ inherently has a "band" of frequencies to which the oscillator can converge (as discussed in [20]). However, given a particular application one needs to find an optimal value of $\omega_{0}$ for each of the oscillators so that the time to converge to the required frequency satisfies the application constraint.

After frequency convergence of the oscillators, the slope of the variable $V_{A}$ in (21) represents the relative amplitude of the adapted frequency component. Fig. 10 shows the simulation results of $A$ for monotone signals with the same frequency $(5 \mathrm{~Hz})$ but with different amplitudes. It can be seen that the slopes of the graphs, after the oscillator converge to the input frequency are proportional to the amplitude of the input, as has been discussed in Section III-A, and thus actually corresponds to the relative amplitude of the input frequency component.

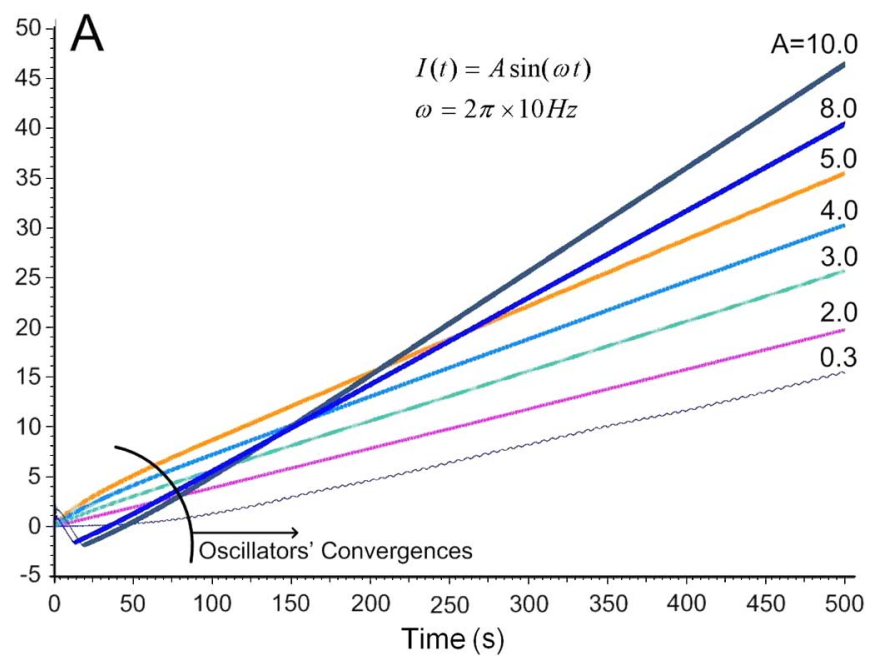

Fig. 10. A slopes for different amplitude inputs after frequency convergence of the oscillator.

\section{Circuit REAlization}

\section{A. Equivalent Circuit Realization}

From Section III-A and the equations therein [(21)], it is evident that there is a self-limitation mechanism which restricts the energy of the system (or the amplitude of the oscillation) to grow beyond a certain level dictated by the value of $\mu$. This feature, which is consistent with (3), is obeyed by a conventional oscillator intrinsically since its oscillation amplitude is restricted in practice by the supply voltage. Taking this fact into account, the first two equations of (21) can be rewritten as follows:

$$
\begin{aligned}
& \dot{V}_{x}=\overbrace{\left(\mu-\left(\left|V_{x}\right|+\left|V_{y}\right|\right)\right) \operatorname{sgn}\left(V_{x}\right)+\omega_{0} V_{y}}+V_{\Delta \omega} V_{y} \\
& \dot{V}_{y}=\underbrace{\left(\mu-\left(\left|V_{x}\right|+\left|V_{y}\right|\right)\right) \operatorname{sgn}\left(V_{y}\right)-\omega_{0} V_{x}}_{\text {Conventional Oscillator }}-V_{\Delta \omega} V_{x}
\end{aligned}
$$




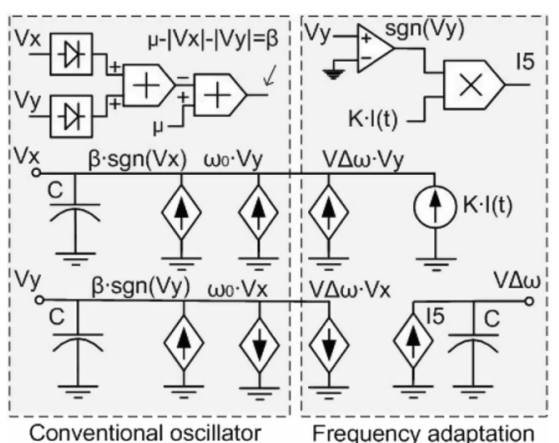

(a)

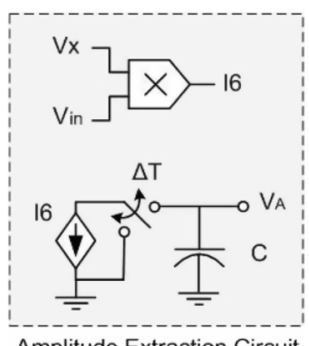

Amplitude Extraction Circuit

(b)
Fig. 11. Equivalent circuit of the oscillator. (a) Oscillator and (b) amplitude extraction.

where the first part of the equation would be considered as a conventional oscillator with the intrinsic frequency $\omega_{0}$ and the second part represents the frequency variation $\Delta \omega$, which is governed by the $V_{\Delta \omega}$ adaptation equation in (21). Depending on the circuit design parameters, $V_{\Delta \omega}$ varies in the range of $V_{\min } \leq V_{\Delta \omega} \leq V_{\max }$, which corresponds to the variation of oscillation frequency in the range of $\omega_{\min } \leq \omega_{\mathrm{osc}} \leq \omega_{\max }$, in other words

$$
\Delta \omega=\omega_{\max }-\omega_{\min }
$$

A simple implementation of such an oscillator can be a ring oscillator with controllable loop delay [25].

Accordingly, one may represent (22) in the form of an equivalent circuit as shown in Fig. 11(a), where the associated parameters are already defined in (21). The equivalent circuit consists of a number of controlled current sources and a multiplier and two adders. The important point is that the learning function can be directly represented by a full-wave rectifier only, which is extremely simple to realize in practice. To compute the amplitude of the adapted frequency component, as mentioned earlier, one needs an integrator with a controlled current source. This current is generated by multiplying the value of $V_{x}$ with the input. However, as mentioned in the previous section, the integration operation needs to be stopped as soon as the oscillator converges to the target frequency. This is shown in Fig. 11(b), where $\Delta T$ represents the integration time.

\section{B. Physical Circuit Realization}

The block diagram of the circuit implementation of the proposed adaptive oscillator following the equivalent circuit depicted in Fig. 11 is shown in Fig. 12, where the main components consist of two analogue multipliers, a state voltage where we are holding the value of $\omega$ and a frequency variable oscillator. The analogue multipliers allow the arithmetic combination of the input signal $V_{i n}$ and the feedback signal $V_{y}$ in order to obtain the amplitude of the input voltage $V_{i n}$ and to establish the value of $\omega$ as explained in Sections III-E and III-F. The state variables $V_{x}$ and $V_{y}$, of Fig. 12, are represented as the voltages at the internal nodes of the tunable oscillator whereas the coupling constant $\mathrm{K}$ is represented as a gain factor in the multiplier block. Similarly $K_{A}$ is implicitly reflected in the amplitude evaluation block of Fig. 12 and here considered to be 1 .

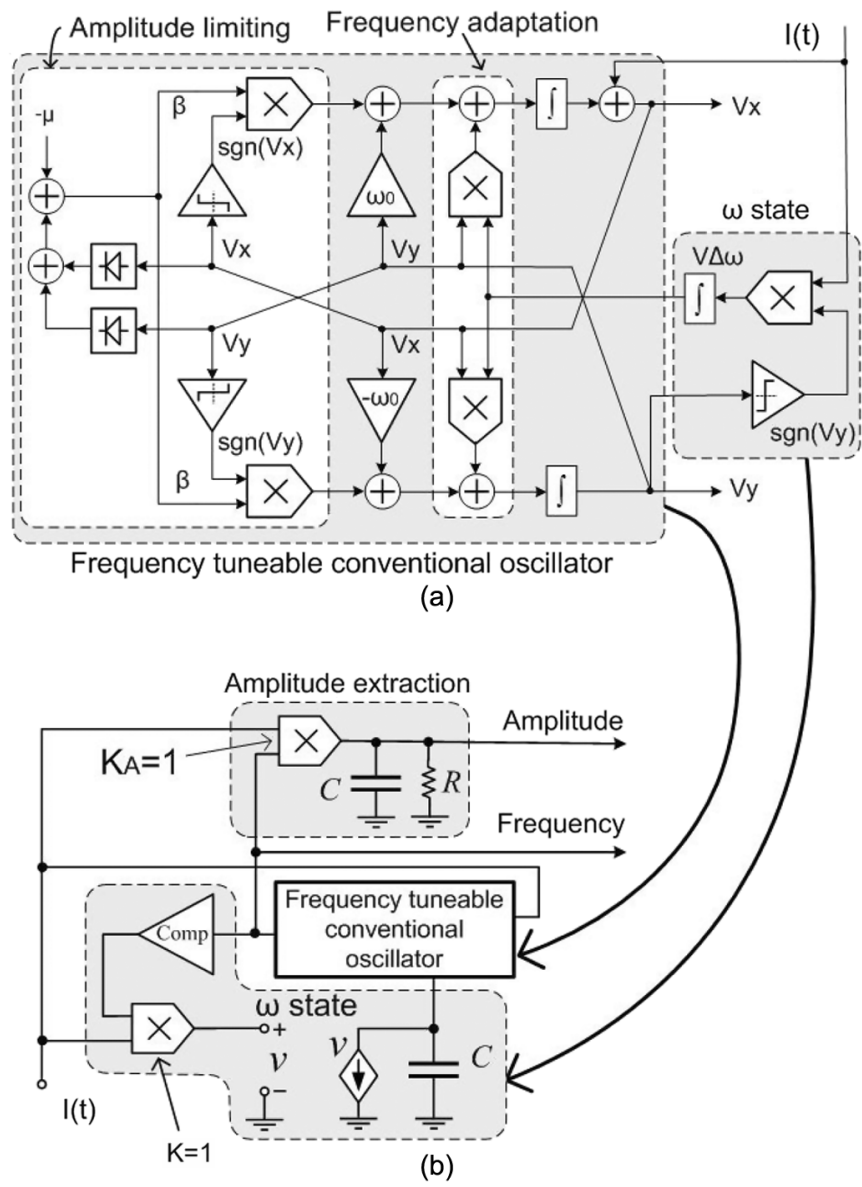

Fig. 12. Block diagram of the oscillator. (a) Block diagram implementation of the equivalent circuit of Fig. 11. (b) Implementation based on conventional oscillator.

Fig. 13(a) and (b) shows a transistor-level circuit implementation of the proposed adaptive oscillator and the amplitude extraction circuitry which directly follows from its block diagram in Fig. 12. The circuit is designed using $120 \mathrm{~nm}$ technology with a dual ended supply of $\pm 0.6 \mathrm{~V}$ and threshold voltage $\approx 230 \mathrm{mV}$.

Overall, the circuit can be divided into two major parts. The first part does the frequency adaptation of the incoming signal; while the second part calculates the amplitude of the input frequency component. The former, itself is divided into five subcircuits, as shown in Fig. 13: a multiplier core, a current subtracting circuit, a current-to-voltage converter, a state voltage (where we calculate the value of $\omega$ ), and a tuneable oscillator. The multiplier core, the current subtracting circuit and the current-to-voltage converter together make the multiplier block as shown in Fig. 13. The circuit used for extracting the amplitude of the input signal also consists of a multiplier core, a current subtracting circuit and a current-to-voltage converter.

The multiplier core (transistors M1-M11 in Fig. 13) is based on the CMOS Gilbert cell [26], where all the transistors operate in the saturation region. The current subtracting circuit is given by transistors M12-M17. These perform subtraction of the currents flowing at the source nodes of the transistors M1 and M2 respectively. The current to voltage converter is a basic 2-stage operational amplifier set as a current to voltage converter by 


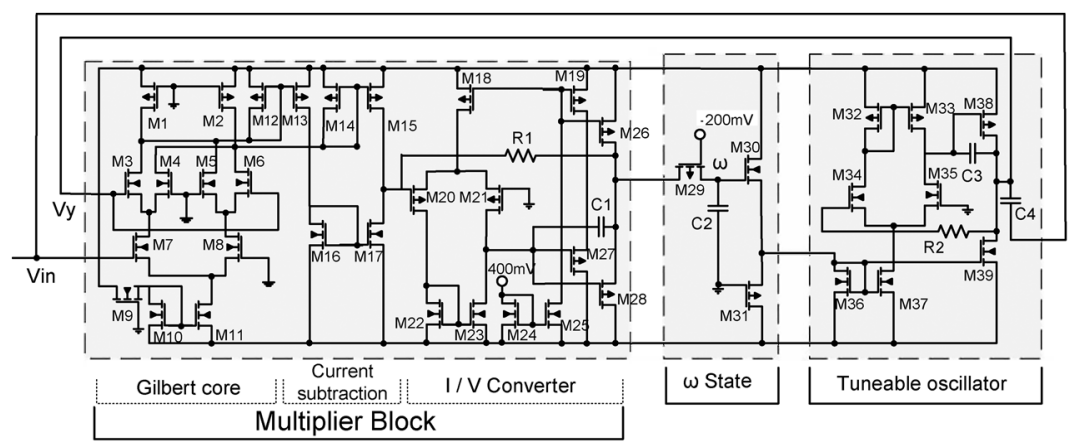

(a)

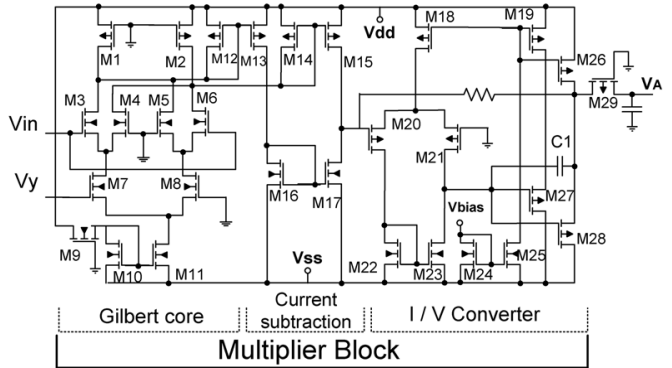

(b)

Fig. 13. Transistor level implementation of the system using traditional circuit elements. (a) Adaptive oscillator. (b) Amplitude measurement.

transistors M18-M28. Furthermore, we can adjust the resistor $\mathrm{R} 1$ of the current-to-voltage converter in order to increase the gain $\mathrm{K}(\mathrm{K}=1)$. The $\omega$ state, which is corresponding with the input frequency of the signal, is given by the transistor M29 (W $=0.15 \mu \mathrm{m}, \mathrm{L}=0.12 \mu \mathrm{m}$ ) set as a resistor with voltage bias equal to $-200 \mathrm{mV}$ and capacitor $\mathrm{C} 2(5 \mathrm{pF})$. The $\mathrm{RC}$ combination of transistor M29 and the capacitor $\mathrm{C} 2$ creates a delay in the feedback loop which allows adaptation of the frequency in the range of 7-45 MHz. Therefore, different bandwidths can be chosen depending on the application by changing either the dimensions of M29, the bias voltage or the value of the capacitor $\mathrm{C} 2$.

As discussed and shown in (22), in this implementation approach the amplitude limitation mechanism of the Hopf oscillator is embedded in a tuneable oscillator. The tuneable oscillator (transistors M29-M38 in Fig. 13) is based on a source follower and a dual stage NMOS based operational amplifier which allows high frequency bandwidth in the $\mathrm{MHz}$ range. The role of the source follower is to transfer the voltage value of $\omega$ to the current source of the amplifier without influencing the $\omega$ state voltage explained before. Indeed, the voltage value of $\omega$ is used to increase or decrease the current given by the transistors M35-M36 and therefore to vary the frequency response of this tuneable oscillator. The negative input of the amplifier (M31-M38) is influenced by the input signal through the capacitor $\mathrm{C} 4(1 \mathrm{pF})$ and connected to its negative input at the gate of transistor M33 while the positive input node is held to ground. Note that in this circuit we use a very well-known configuration for the tuneable oscillator as a standard controllable delay-line oscillator. As mentioned earlier, any configuration for the core oscillator is possible depending on the input frequency spectrum and the required performance criteria. The particular configuration described here is only for the sake of an example. All the transistor dimensions of the complete circuit are given in Table II.

The circuit used to detect the amplitude of the incoming signal uses the same subcircuits explained in this section above, where the input $\mathrm{V}_{\text {in }}$ of the multiplier core is connected to the input signal and the second input $\mathrm{V}_{y}$ of the multiplier core is connected to the output of the tuneable oscillator as described by (21), capturing the amplitude of the signal.
TABLE II

TRANSISTOR DIMENSIONS USED FOR THE CIRCUIT LEVEL IMPLEMENTATION

\begin{tabular}{c||l|l||c||l|l}
\hline \hline$\overline{\text { Transistor }}$ & W/L & W $(\mu \mathbf{m})$ & Transistor & W/L & W $(\mu \mathbf{m})$ \\
\hline \hline M1-M2 & 8.3 & 1.0 & M27 & 1.58 & 0.19 \\
\hline M3-M6 & 33.0 & 4.0 & M28 & 16.67 & 2.0 \\
\hline M7-M8 & 4.2 & 0.5 & M29 & 1.25 & 0.15 \\
\hline M9-M11 & 3.75 & 0.45 & M30 & 166.67 & 20.0 \\
\hline M12-M15 & 1.25 & 0.15 & M31 & 4.2 & 0.5 \\
\hline M16-M17 & 1.25 & 0.15 & M32-M33 & 8.3 & 1.0 \\
\hline M18-M19 & 1.25 & 0.15 & M34-M35 & 3.25 & 0.39 \\
\hline M20-M21 & 333.33 & 40.0 & M36 & 1.25 & 0.15 \\
\hline M22-M23 & 5.42 & 0.65 & M37 & 5.0 & 0.6 \\
\hline M24-M25 & 4.2 & 0.5 & M38 & 166.67 & 20.0 \\
\hline M26 & 1250 & 150 & M39 & 20.0 & 2.4 \\
\hline \hline
\end{tabular}

\section{Simulation Results}

A full set of simulations was performed to evaluate the circuit performance by Cadence Spectre as presented in the following subsections. In all these simulations, according to (21) the $y$-axes are the value of the state variable representing the frequency in volts.

\section{A. Adaptation to Monotone and Multiple-Frequency Signals}

Fig. 14 shows response of the circuit to a monotone sinusoidal input. It can be observed that the circuit level behavior of the oscillator is consistent with the system level simulation (shown in Fig. 7). As discussed before, there is a frequency basin corresponding to the value of the intrinsic frequency of each oscillator which indicates the range of the frequencies that can be adapted by the oscillator. In this particular case the frequency basin ranges from 7 to $45 \mathrm{MHz}$. However it can be seen from Fig. 14 that mapping of the detected frequencies to this range is not linear. This nonlinearity is quite prominent at the lower and upper ends of the frequency basin while maintaining linear mapping around the middle range. On the contrary, the system level simulation in Fig. 7 showed that a linear mapping relationship exists throughout the range of frequency basin. This nonlinear mapping effect is attributed to the saturation effect of the transistor components and its subsequent effect on the oscillator behavior and its controlling mechanism close to the boundaries of the frequency basin. 


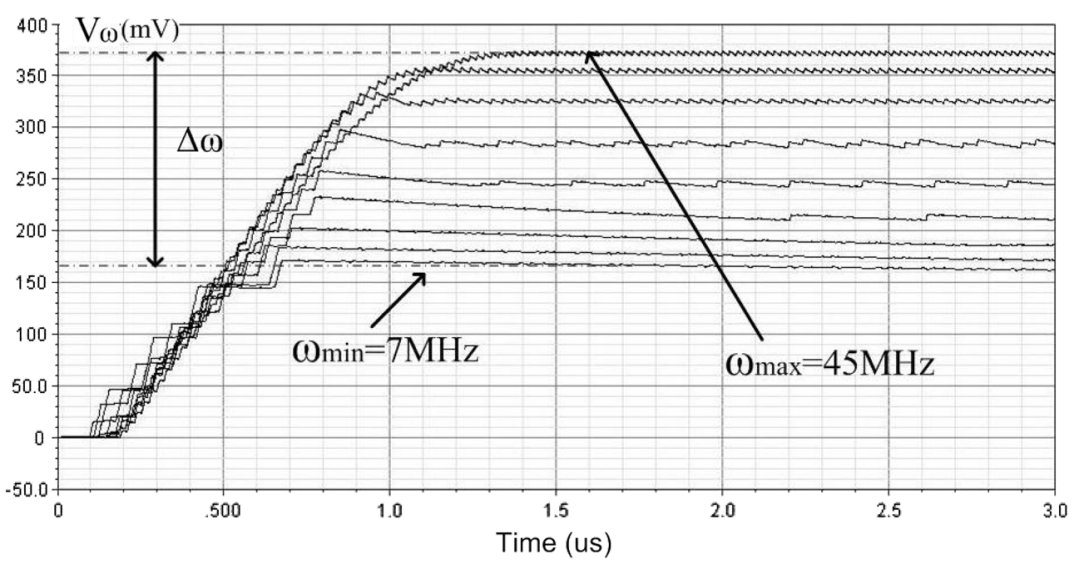

Fig. 14. Circuit response to different input frequencies over the frequency catchment range $\left(\left[\omega_{\min }, \omega_{\max }\right]\right)$.

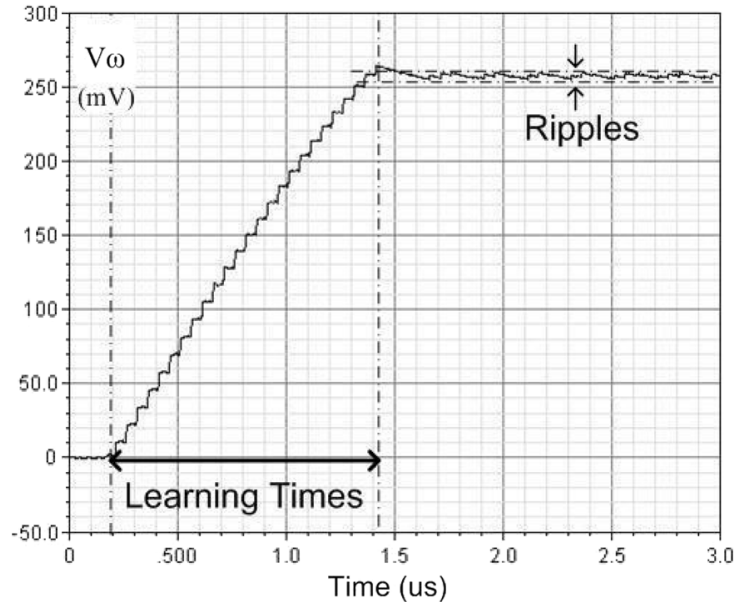

Fig. 15. Oscillator response to $I(t)=10 \sin \left(1 \times 10^{6} t\right)+50 \sin (20 \times$ $\left.10^{6} t\right)+10 \sin \left(10 \times 10^{6} t\right) \mathrm{mV}$.

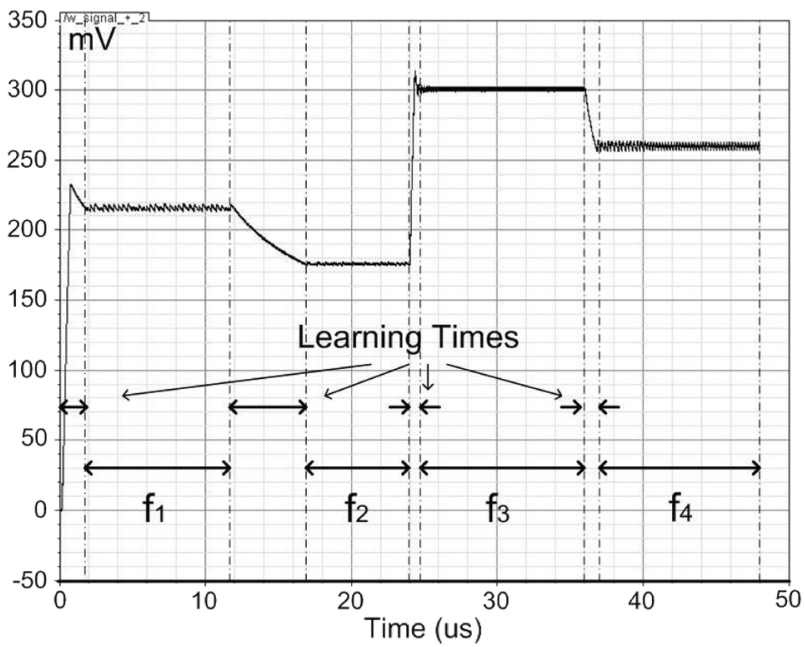

Fig. 16. Behavior of the oscillator under nonstationary monotone.

Simulation results for a multifrequency input signal are presented in Fig. 15, which shows that after a certain learning time the oscillator adapts to the input frequency component having the largest amplitude. In both results of Figs. 14 and 15, there exists a small ripple about the converged frequency value in the steady state. According to the results of our simulations, amplitude of this ripple is directly related to the amplitude of the frequency components in the input. Referring to the oscillator equation (see (17) for instance) the input signal is directly added to the $x$ equation and it is expected in the output oscillation.

\section{B. Nonstationary Signals}

The response of the oscillator to a nonstationary input is depicted in Fig. 16, where the input signal is composed of different frequencies at different times instants. As can be observed, the oscillator adapts its frequency to the new input frequency in each period of time. The time required to adapt to a new frequency ("learning time") depends strongly on the difference between the already adapted frequency and the frequency to be adapted and thus may be different in different time instants. However the result is consistent with the system level simulation and is as expected from the mathematical property of the adaptive oscillator. It is to be noted that unlike system-level simulations, the learning times at the circuit level are very short and even negligible in most of the cases.

The frequency adaptation characteristic of the proposed adaptive oscillator is further studied using a real-life EMG signal captured from human subject. The result is shown in Fig. 17 where the captured EMG signal is shown at the upper left side of Fig. 17. The frequency content of the EMG signal is analyzed by performing 256-point FFT in Matlab as shown on the upper right side of Fig. 17. It is evident from the Fourier spectrum that the dominant frequency component present in this case is $40 \mathrm{~Hz}$. In this simulation, we have employed only one low frequency oscillator and thereby expect it to get tuned with this dominant frequency component only. The frequency adaptation characteristic of the proposed circuit is shown in the lower part of Fig. 17. It is clearly evident that the behavior of the oscillator is exactly as expected.

\section{Amplitude Detection}

The simulation characteristic of the proposed adaptive oscillator for detecting amplitudes of different frequency components is shown in Fig. 18. In this simulation, several inputs with different amplitude and same frequencies are applied to the circuit. It is clearly evident from Fig. 18 that there exists a one-to-one relationship of the amplitude of the applied 

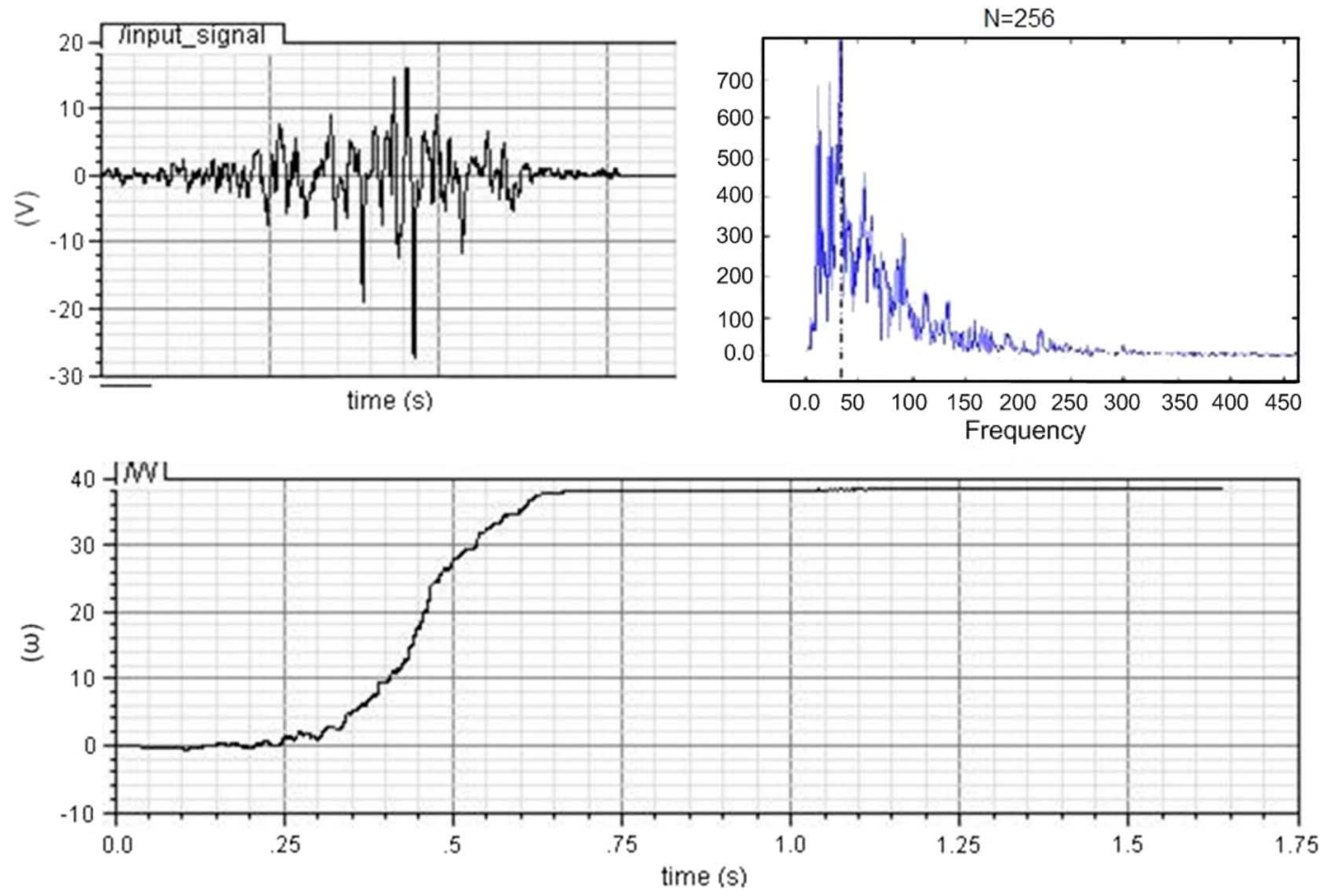

Fig. 17. Oscillator response to an EMG signal.

frequency components and the corresponding output voltage. However, as in the case of frequency adaptation, there exists some nonlinearity in this relationship around the edge of the frequency basin.

\section{GENERAL Discussion AND OPEN QUESTIONS}

Although our design methodology for VLSI realizable Hopf oscillator with dynamic plasticity and corresponding simulation results show good promise for applying such system in signal processing, there are issues which require more investigation in future studies. First of all, as it is attainable from the simulations results, in real-life circuit, the oscillator maps frequency and amplitude values to the voltage space nonlinearly in contrast to the system level behavior. This nonlinearity is more observable when the input frequency is closer to the edge of the frequency basin which we believe is the result of nonlinear behavior of the tuneable oscillator at the boundaries of the frequency basin. This issue and its relationship with the frequency resolution of the oscillator need more investigation in future. Secondly, the ripple values of the frequency state after convergence and its relationship with the input perturbation amplitude and frequency is another matter of concern. Overall, the ripple value is directly dependent on the perturbation amplitude but this dependency needs to be formulated, in terms of the frequency spectrum, using different types of inputs. Thirdly, at present, the proposed oscillator is only capable of adapting its frequency to the dominant frequency component in the frequency spectrum of the input signal. A suitable method needs to be formulated for adapting its frequency to any arbitrary frequency components of the input spectrum as long as they fall within its frequency basin. This particular problem leads to the fourth open question - the resolution of the oscillator given a

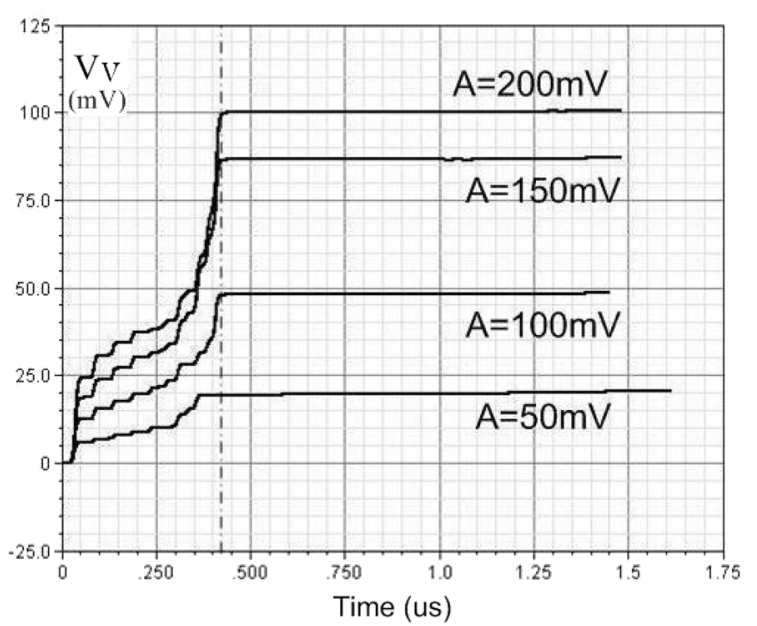

Fig. 18. Simulated characteristics of amplitude detection circuit.

particular intrinsic frequency and also quantification of learning time of the oscillator. Another important question is how to realize an equivalent digital implementation of the proposed oscillator and by doing this whether there exists any advantage in terms of silicon area and power consumption compared to the analog counterpart. Currently these open questions are under investigation and will be addressed in our future works. These investigations are particularly important from the viewpoint of deploying a population of such adaptive oscillators in hardware for general purpose signal analysis at the cost of very small silicon area and power dissipation.

\section{CONCLUSION}

In this paper, we present a new VLSI realizable nonlinear Hopf oscillator with dynamic plasticity using the analog design approach for the first time. The proposed oscillator shows the 
enhanced frequency basin compared to the theoretical ones existing in literature. Our results show that this oscillator learns the frequency of the input signal and also does so in a shorter time. The circuit simulation results show consistency with the mathematical model. In addition, the method and subsequent circuit proposed for amplitude extraction of the frequency components implies that it is possible to employ the proposed oscillator for performing a wide range of signal processing tasks on real-life hardware. But there are several open questions which are currently under investigation. However, despite all these open questions, due to its compact analog implementation the oscillator shows an immense potential to be used in analog frequency analysis method and thereby paving its way to realize a unified analog Fourier and Wavelet transform processor.

\section{REFERENCES}

[1] ITRS [Online]. Available: http://public.itrs.net

[2] N. R. Shanbhag, S. Mitra, G. de Veciana, M. Orshansky, R. Marculescu, J. Roychowdhury, D. Jones, and J. M. Rabaey, "The search for alternative computational paradigms," IEEE Design \& Test of Comput., vol. 25, no. 4, pp. 334-343, July-August 2008.

[3] E. Izhikevich and Y. Kuramoto, "Weakly coupled oscillators," in Encyclopedia of Mathematical Physics. New York: Academic, 2006, p. 5:448.

[4] J. J. Collins and I. Stewart, "Hexapodal gaits and coupled nonlinear oscillator models," Biolog. Cybern., vol. 68, no. 4, pp. 287-298, Feb. 1993.

[5] M. Golubitsky, I. Stewart, P.-L. Buono, and J. J. Collinsc, "A modular network for legged locomotion," Physica D, vol. 115, no. 1-2, pp. 56-72, 1998.

[6] R. Borisyuk, M. Denham, F. Hoppensteadt, Y. Kazanovich, and O. Vinogradova, "Oscillatory model of novelty detection," Network: Comput. Neural Syst., vol. 12, no. 1, pp. 1-20, Feb. 2001.

[7] T. Nishikawaa, F. C. Hoppensteadtb, and Y.-C. Lai, "Oscillatory associative memory network with perfect retrieval," Physica D: Nonlin. Phenom., vol. 197, no. 1-2, pp. 134-148, Oct. 2004.

[8] D. Eckc, "Finding downbeats with a relaxation oscillator," Psycholog. Res., vol. 66, pp. 18-25, 2001.

[9] E. W. Large and J. F. Kolen, "Accent structures in music performance," Connect. Sci., vol. 6, pp. 177-208, 1994.

[10] L. Chua and T. Roska, Cellular Neural Networks and Visual Computing: Foundations and Applications. Cambridge, U.K.: Cambridge University Press, 2005.

[11] I. Szatmári and L. O. Chua, "Awakening dynamics via passive coupling and synchronization mechanism in oscillatory cellular neural/nonlinear networks," Int. J. Circuit Theory and Applic., vol. 36, no. 5-6, pp. 525-553, Jun. 2008.

[12] K. Chen and D. Wang, "A dynamically coupled neural oscillator network for image segmentation," Neur. Netw., vol. 15, no. 3, pp. 423-439, Apr. 2002

[13] D. Xu, J. C. Principe, and J. G. Harris, "Logic computation using coupled neural oscillators," in Proc. Int. Symp. Circuits and Systems (ISCAS), May 2004, vol. 5, pp. V-788-V-791, IEEE.

[14] C. M. Pinto and M. Golubitsky, "Central pattern generators for bipedal locomotion," J. Mathemat. Biol., vol. 53, no. 3, pp. 474-489, Sep. 2006.

[15] J. Buchli and A. J. Ijspeert, "A simple, adaptive locomotion toy-system," in Proc. SAB'04, 2004, pp. 153-162.

[16] J. Buchli, L. Righetti, and A. J. Ijspeert, "Frequency analysis with coupled nonlinear oscillators," Physica D: Nonlin. Phenom., vol. 237, no. 13, pp. 1705-1718, Aug. 2008.

[17] P. G. Drazin, Nonlinear Systems (Cambridge Texts in Applied Mathematics). Cambridge, U.K.: Cambridge University Press, Jan. 2008.

[18] A. Ahmadi, E. Mangieri, K. Maharatna, and M. Zwolinski, "Physical realizable circuit structure for adaptive frequency hopf oscillator," in NEWCAS-TAISA'09: Joint IEEE North-East Workshop on Circuits and Systems and TAISA Conf., Jun. 2009, vol. 1, pp. 1-4.

[19] A. I. Mees and L. Chuac, "The Hopf bifurcation theorem and its applications to nonlinear oscillations in circuits and systems," IEEE Trans. Circuits Syst., vol. CAS-26, no. 4, pp. 235-254, Apr. 1979.
[20] L. Righetti, J. Buchli, and A. J. Ijspeert, "Dynamic hebbian learning in adaptive frequency oscillators," Physica D: Nonlin. Phenom., vol. 216, no. 2, pp. 269-281, 2006.

[21] H. K. Khalil, Nonlinear Systems, 3rd ed. Upper Saddle River, NJ: Prentice-Hall, Jan. 2002.

[22] A. V. Oppenheim, R. W. Schafer, and J. R. Buck, Discrete-Time Signal Processing. : Pearson U.S. Imports and PHIPEs, 1999.

[23] J. G. Proakis, Digital Signal Processing. Upper Saddle River, NJ: Prentice-Hall, 2006.

[24] P. J. Ashenden, G. D. Peterson, and D. A. Teegarden, The System Designer's Guide to VHDL-AMS: Analog, Mixed-Signal, and Mixed-Technology Modeling (Systems on Silicon). New York: Morgan Kaufmann, Sep. 2003.

[25] S. Joeres, A. Kruth, O. Meike, G. Ordu, S. Sappok, R. Wunderlich, and $\mathrm{S}$. Heinen, "Design of a ring-oscillator with a wide tuning rangein 0.13 $\mu \mathrm{m}$ CMOS for the use in global navigation satellite systems," in Proc. ProRISC, 2004, pp. 529-535.

[26] B. Razavi, Fundamentals of Microelectronics. New York: Wiley, 2008.

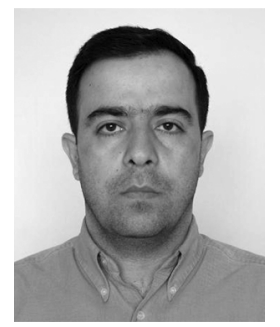

Arash Ahmadi (M'04) received the B.Sc. degree from Sharif University of Technology, Tehran, Iran, in 1993 and the M.Sc. degree from Tarbiat Modares University, Tehran, Iran, in 1997, both in electronics engineering, and the Ph.D. degree in electronics from the University of Southampton, Southampton, U.K., in 2008.

From 2008 to 2010, he was a Fellow Researcher with the School of Electronics and Computer Science, University of Southampton. He is currently an Assistant Professor in the Electronic Department, Razi University, Kermanshah, Iran. His current research interests include high-level synthesis, digital circuit design and optimization and bio-inspired computing.

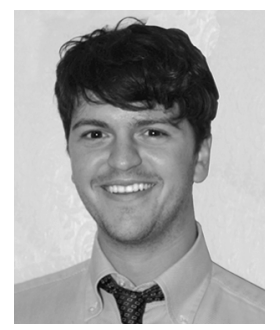

Eduardo Mangieri was born in Rome, Italy, in 1982. He received the B.Eng. degree in electronics engineering from the University of Hull, Hull, U.K., in 2005, the M.S. degree (on full scholarship) in microelectronics from the University of Newcastle, Newcastle, U.K., in 2006, and the M.Sc. degree in system on chip (SoC) in 2007 from the University of Southampton, Southampton, U.K., where he is currently pursuing the Ph.D. degree in analog electronics and biomedical signals..

His research interests involve $\mathrm{SoC}$, analogue and digital VLSI, DSP, low-power and compact design, neural networks, brain studies, bio-medical signals such as electrocardiogram (ECG) and electromyography (EMG) signals, and medical devices.

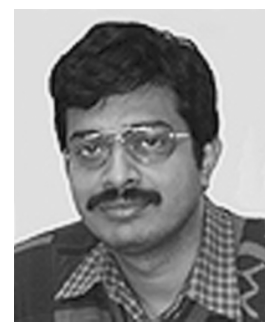

Koushik Maharatna (M'02) received the M.Sc. degree in electronic science from Calcutta University, Calcutta, India, in 1995 and the Ph.D. degree from Jadavpur University, Calcutta, in 2002.

From 1996 to 2000, he was involved in projects sponsored by the Government of India undertaken at the Indian Institute of Technology (IIT), Kharagpur, India. From 2000 to 2003, he was a Research Scientist with IHP, Frankfurt (Oder), Germany. During this phase, his main involvement was related to the design of a single-chip modem for the IEEE 802.11a standard. In September 2006, he joined the Electronic Systems and Devices Group at the School of Electronics and Computer Science, University of Southampton, Southampton, U.K., where he is currently a Senior Lecturer. His research interests include low-power VLSI and signal processing for applications in DSP, communication and next-generation healthcare systems, computer arithmetic, analog signal processing, and bio-inspired circuits and systems.

Dr. Maharatna is a member of the Engineering and Physical Research Council (EPSRC) College in the U.K and of the IEEE VLSI System Application (VSA) Technical Committee. 


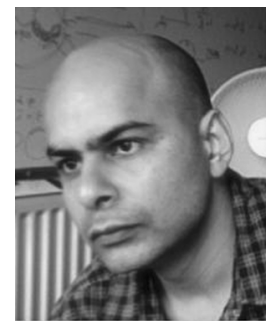

Srinandan Dasmahapatra received the B.Sc. degree from Calcutta University, Calcutta, India, in 1986 and the Ph.D. degree in physics from SUNY, Stony Brook, NY, in 1992.

$\mathrm{He}$ is currently a Lecturer in the School of Electronics and Computer Science, University of Southampton , Southampton, U.K. His research interests include artificial intelligence and pattern recognition, probabilistic knowledge representation, and more recently, systems biology.

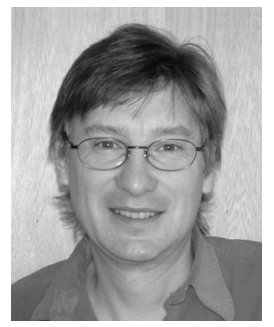

Mark Zwolinski (M'92-SM'00) received the B.Sc. and Ph.D. degrees from the University of Southampton, Southampton, U.K., in 1982 and 1986, respectively.

From 1990 to 2005, he was a Lecturer, Senior Lecturer, and Reader in the School of Electronics and Computer Science, University of Southampton, and since 2005, he has been a Full Professor. His current research interests include electronic design automation, low-power and reliable design, and multicore platforms. He has published over 140 refereed journal and conference papers and is the author of three books.

Dr. Zwolinski has served on the Technical Program Committees of many conferences including DAC, DATE, and ETS, and was the Technical Program Chair for ICECS 2007. He is a Fellow of the IET and of BCS and a Senior Member of ACM. 